\title{
Clinical relevance of aortic calcification in urolithiasis patients
}

Toshikazu Tanaka', Shingo Hatakeyama ${ }^{1 *}$ D, Hayato Yamamoto', Takuma Narita', Itsuto Hamano', Teppei Matsumoto', Osamu Soma', Yuki Tobisawa', Tohru Yoneyama², Takahiro Yoneyama', Yasuhiro Hashimoto², Takuya Koie ${ }^{1}$, Ippei Takahashi ${ }^{4}$, Shigeyuki Nakaji ${ }^{4}$, Yuriko Terayama ${ }^{3}$, Tomihisa Funyu ${ }^{3}$ and Chikara Ohyama ${ }^{1,2}$

\begin{abstract}
Background: The aim of the present study is to investigate the clinical relevance of aortic calcification in urolithiasis patients.

Methods: Between January 2010 and September 2014, 1221 patients with urolithiasis were treated in Oyokyo Kidney Research Institute and Hirosaki University Hospital. Among these, 287 patients (Stone group) on whom adequate data were available were included in this retrospective study. We also selected 148 subjects with early stage (pT1NOMO) renal cell carcinoma from 607 renal cell carcinoma patients who underwent radical nephrectomy at Hirosaki University Hospital (Non-stone group) as control subjects. Validity of the Non-stone group was evaluated by comparison with pair-matched 296 volunteers from 1166 subjects who participated in the Iwaki Health Promotion Project in 2014. Thereafter, age, body mass index, aortic calcification index (ACl), renal function, serum uric acid concentrations, and comorbidities (diabetes, hypertension, or cardiovascular disease) were compared between the Non-stone and Stone groups. Independent factors for higher ACl and impaired renal function were assessed using multivariate logistic regression analysis.

Results: We confirmed relevance of Non-stone group patients as a control subject by comparing the pairmatched community-dwelling volunteers. Backgrounds of patients between the Non-stone and Stone groups were not significantly different except for the presence of hypertension in the Stone group. ACl was not significantly high in the Stone group compared with the Non-stone group. However, age-adjusted ACl was greater in the Stone group than the Non-stone group. Among urolithiasis patients, ACl was significantly higher in uric acid containing stone patients. The number of patients with stage 3B chronic kidney disease (CKD) was significantly higher in the Stone group than in the Non-stone group (12\% vs. 4\%, $P=0.008$ ). Multivariate logistic regression analysis showed higher aortic calcification index (>13\%), and being a stone former were independent factors for stage 3B CKD at the time of diagnosis.
\end{abstract}

Conclusion: Aortic calcification and being a stone former had harmful influence on renal function. This study was registered as a clinical trial: UMIN: UMIN000022962.

Keywords: Urolithiasis, Stone former, Aortic calcification, Chronic kidney disease, Renal function

\footnotetext{
* Correspondence: shingoh@hirosaki-u.ac.jp

${ }^{1}$ Department of Urology, Hirosaki University Graduate School of Medicine, 5

Zaifu-chou, Hirosaki 036-8562, Japan

Full list of author information is available at the end of the article
} 


\section{Background}

Urolithiasis is a common urological disease, and its prevalence has been increasing in Japan similar to that in other developed countries [1]. In 2005, in Japan, the age-standardized annual incidences of the first episode of upper tract stones were reported to be $165.1 / 100,000$ men and 65.1/100,000 women, which were 2-fold higher compared with that in 1965 [2]. During this period, lifestyle and dietary habits in Japan were more westernized, and the prevalence of obesity and metabolic syndrome (MetS) increased rapidly [3]. MetS refers to a cluster of risk factors, including high blood pressure, obesity, high cholesterol, type 2 diabetes, and atherosclerotic cardiovascular disease [4]. Moreover, many epidemiological studies have suggested an association among urolithiasis, MetS, and chronic kidney disease (CKD) [5-7]. Because urolithiasis is considered as one of the consequences of MetS, we hypothesized that concurrent aortic calcification, complicated by arterial stiffness and atherosclerosis, may be responsible for developing CKD. Aortic calcification has recently been considered as a major complication and an independent risk factor for CKD, coronary diseases, heart failure, and stroke [8-10]. Aortic calcification is widely used as an indicator of MetS-related disease [11], and it can be quantitatively measured by the aortic calcification index (ACI) using abdominal computed tomography (CT). We have previously reported the clinical relevance of ACI in hemodialysis patients, renal transplant recipients, and primary aldosteronism patients [12-14]. However, only a few studies have investigated aortic calcification and urolithiasis [15], and the implication of aortic calcification in urolithiasis patients remains unclear. In the present study, we retrospectively assessed the distribution of aortic calcification, and influence of aortic calcification on renal function in urolithiasis patients. This study was registered as a clinical trial: UMIN000022962.

\section{Methods}

Between January 2010 and September 2014, we treated 1221 stone patients with urolithiasis in Oyokyo Kidney Research Institute and Hirosaki University Hospital. We excluded the patients whose data including stone information and blood exam were inadequate. As a result, the remaining 287 patients (Stone group) who underwent pre-treatment abdominal CT and laboratory testing such as renal function, uric acid, lipid metabolism, and urinalysis were included in this retrospective study (Fig. 1a). The non-stone subjects comprised renal cell carcinoma (RCC) patients who had undergone radical nephrectomy at Hirosaki University Hospital between December 1986 and March 2015. Because pre-treatment abdominal CT and laboratory testing were necessary in those patients, we selected early stage RCC patients as a control group. Of 607 RCC patients, 148 early stage RCC patients (pT1N0M0) were selected as the nonstone control subjects (Non-stone group). Laboratory testing was performed before stone treatment or radical nephrectomy. The estimated glomerular filtration rate (eGFR) was calculated using the Modification of Diet in Renal Disease equation for Japanese patients [16].

To guarantee the validity of early stage RCC patients, we employed pair-matched 296 community-dwelling volunteers from 1166 subjects who participated in the Iwaki Health Promotion Project in 2014 [17]. The demographic data (age, sex, body mass index) and medical information (positive history of hypertension, cardiovascular disease, diabetes, and dyslipidemia) were obtained from self-questionnaires and interviews in this project. To select appropriate subjects, we used the propensity score matching strategy to compare renal function as described previously [18]. Propensity scores were calculated using logistic analysis, and the data used in the analyses included age, gender, body mass index, positive history of cardiovascular disease, hypertension, and diabetes. Based on the scores, two healthy subjects and one RCC patients with a score within 0.03 were selected as a pair (at a 2:1 ratio), and we compared renal function, serum uric acid concentration and lipid disorder between the volunteers and Nonstone group.

In the Non-stone and Stone groups, ACI was quantitatively measured using abdominal CT images (TSX-301B, Toshiba Medical Systems Corp., Ohtawara, Japan, or CT750HD, GE Healthcare Japan, Tokyo, Japan) above the common iliac artery bifurcation by scanning 10 times at $10-\mathrm{mm}$ intervals, as described previously [12]. ACI (\%) expresses the calcification proportion in $12 \mathrm{sec}-$ tors, and it is calculated as the average value of sections 1-10 (Fig. 1b).

After the validity evaluation of early stage RCC patients for control subjects, we investigated the distribution of ACI in urolithiasis patients. Patients' background including age, sex, body mass index, comorbidities (diabetes, hypertension, or cardiovascular disease), renal function, lipid metabolism, serum uric acid concentrations, voluntary urine protein, and ACI were compared between the Non-stone and Stone groups. Thereafter, the impact of stone disease on renal function were compared between the Non-stone and Stone groups.

\section{Statistical analysis}

The statistical analyses of the clinical data were performed using SPSS v. 22.0 (IBM Corporation, Armonk, NY, USA) and GraphPad Prism v. 5.03 (GraphPad Software, San Diego, CA, USA). Categorical variables were reported as percentages and compared using Fisher's exact test or Chi-square test. Quantitative data were 
a

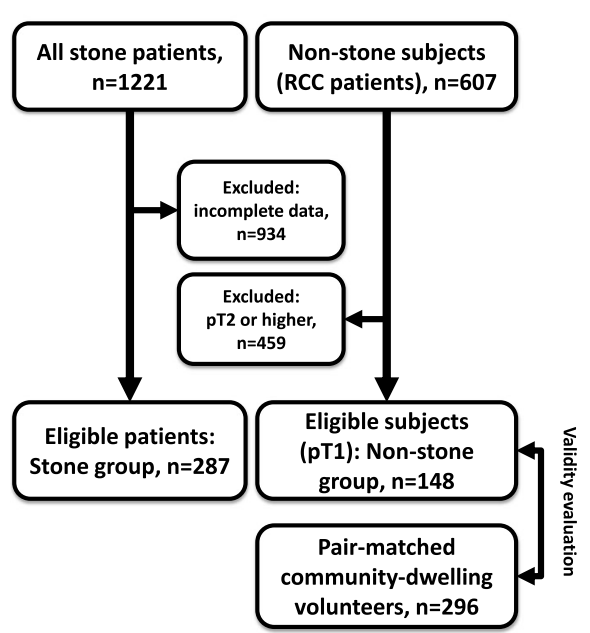

b

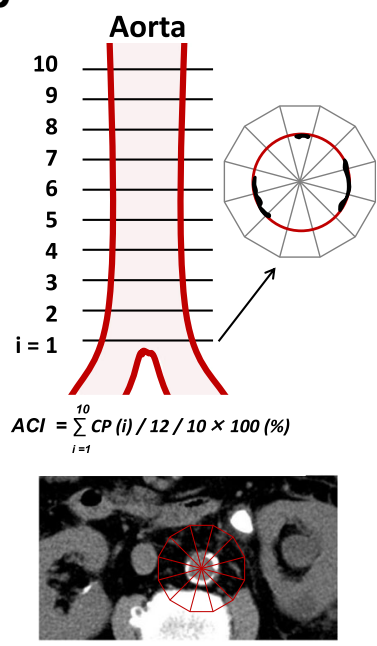

Fig. 1 Patient selection and measurement of aortic calcification index. Eligible stone patients and non-stone subjects were selected from our database in Oyokyo Kidney Research Institute and Hirosaki University Hospital. In the Stone group, 934 patients were excluded because of incomplete data. The non-stone subjects were selected from renal cell carcinoma (RCC) patients who underwent radical nephrectomy at Hirosaki University Hospital. Of those, we selected early-stage RCC patients (pT1NOMO) for the non-stone control subjects (Non-stone group). Validity of the Non-stone group was evaluated by comparison with pair-matched 296 community-dwelling volunteers from 1166 subjects who participated in the Iwaki Health Promotion Project in 2014 (a). Aortic calcification was quantitatively measured using pretreatment abdominal computed tomography images, scanned 10 times at 10-mm intervals above the abdominal aortic bifurcation. The calcification profile was calculated as the sum of calcification areas of 12 fractions in a single slice divided by 12 . The sum of the calcification profile from 10 slices was divided by 10 and multiplied by 100 to obtain the percentage The typical computed tomography (CT) of stone patient is shown. The aortic calcification index (ACI) of this section is $10 / 12 \times 100=83.3 \%$ (b)

expressed as medians with quartiles 1 and 3 (Q1-Q3). Differences between the groups were statistically compared using Student's $t$-test for data with normal distribution or Mann-Whitney $U$-test for data exhibiting a non-normal distribution. Differences among the three groups were statistically compared using Kruskal-Wallis test. When significant differences among the three groups were observed, we performed multiple comparisons. The correlation between two indices was analyzed using Spearman's correlation coefficient. Probability $(P)$ values of $<0.05$ were considered to be statistically significant. The optimal cutoff value for stage 3B CKD was calculated using the following formula [19] $:(1-\text { sensitivity })^{2}+(1-\text { specificity })^{2}$ with the receiver operating characteristic (ROC) curve.

Table 1 Clinical characteristic of community-dwelling volunteers and early stage renal cell carcinoma patients (Non-stone group)

\begin{tabular}{|c|c|c|c|}
\hline & Volunteers & Non-stone group & $P$ value \\
\hline$n$ & 296 & 148 & \\
\hline Age (years) ${ }^{a}$ & $64(56-72)$ & $62(54-72)$ & 0.333 \\
\hline Gender (Male) ${ }^{a}, n=$ & $176(59 \%)$ & $89(60 \%)$ & 0.919 \\
\hline Body mass index ${ }^{a}\left(\mathrm{~kg} / \mathrm{m}^{2}\right)$ & $24 \pm 4$ & $24 \pm 4$ & 0.849 \\
\hline \multicolumn{4}{|l|}{ Comorbidities } \\
\hline Hypertension $^{\mathrm{a}}, n=$ & $85(29 \%)$ & $43(29 \%)$ & 1.000 \\
\hline Diabetes $^{\mathrm{a}}, n=$ & $47(16 \%)$ & $31(21 \%)$ & 0.189 \\
\hline Cardiovascular disease $^{a}, n=$ & $42(14 \%)$ & $20(14 \%)$ & 0.886 \\
\hline eGFR (mL/min/1.73 m²) & $76(66-85)$ & $72(61-87)$ & 0.127 \\
\hline Stage 3B CKD, $n=$ & $12(4 \%)$ & $6(4 \%)$ & 1.000 \\
\hline Uric acid $>7.0 \mathrm{mg} / \mathrm{dL}, n=$ & $34(11 \%)$ & $24(16 \%)$ & 0.180 \\
\hline Dyslipidemia (Total cholesterol $>220$, or Triglyceride $>140 \mathrm{mg} / \mathrm{dL}$ ), $n=$ & $21(7 \%)$ & $15(10 \%)$ & 0.401 \\
\hline Urine protein $>30 \mathrm{mg} / \mathrm{dL}, n=$ & $\mathrm{N} / \mathrm{A}$ & $22(15 \%)$ & \\
\hline Aortic calcification index (ACl) & $\mathrm{N} / \mathrm{A}$ & $6.7(0.8-19.2)$ & \\
\hline
\end{tabular}

Median and interquartile range (Q1-Q3) was used for consecutive variables

${ }^{a}$, applied for propensity score-matching 
Table 2 Patients' characteristics

\begin{tabular}{|c|c|c|c|}
\hline & Non-stone group & Stone group & $P$ value \\
\hline$n$ & 148 & 292 & \\
\hline Age (years) & $62(54-72)$ & $63(54-72)$ & 0.626 \\
\hline Sex (Male), $n=$ & $89(60 \%)$ & 169 (58\%) & 0.683 \\
\hline Body mass index $\left(\mathrm{kg} / \mathrm{m}^{2}\right)$ & $24 \pm 4$ & $25 \pm 4$ & 0.171 \\
\hline \multicolumn{4}{|l|}{ Comorbidities } \\
\hline Hypertension, $n=$ & $43(29 \%)$ & $142(49 \%)$ & $<0.001$ \\
\hline Diabetes, $n=$ & $31(21 \%)$ & $72(25 \%)$ & 0.407 \\
\hline Cardiovascular disease, $n=$ & $20(14 \%)$ & $33(11 \%)$ & 0.536 \\
\hline eGFR $\left(\mathrm{ml} / \mathrm{min} / 1.73 \mathrm{~m}^{2}\right)$ & $72(61-87)$ & $76(58-95)$ & 0.250 \\
\hline Hyperuricemia $(>7.0 \mathrm{mg} / \mathrm{dL}, n=$ & $24(16 \%)$ & $45(15 \%)$ & 0.890 \\
\hline Dyslipidemia (total cholesterol $>220$, or triglyceride $>140 \mathrm{mg} / \mathrm{dL}$ ), $n=$ & $15(10 \%)$ & $31(11 \%)$ & 1.000 \\
\hline Voluntary urine protein $>30 \mathrm{mg} / \mathrm{dL}, n=$ & $22(15 \%)$ & $57(20 \%)$ & 0.240 \\
\hline \multicolumn{4}{|l|}{ Type of stone } \\
\hline Uric acid stone, $n=$ & & $32(11 \%)$ & \\
\hline Non-uric acid stone, $n=$ & & $216(74 \%)$ & \\
\hline Unknown, $n=$ & & $44(15 \%)$ & \\
\hline Aortic calcification index (ACl) & $6.7(0.8-19.2)$ & $7.1(0.8-22.5)$ & 0.856 \\
\hline
\end{tabular}

Median and interquartile range (Q1, Q3) was used for consecutive variables
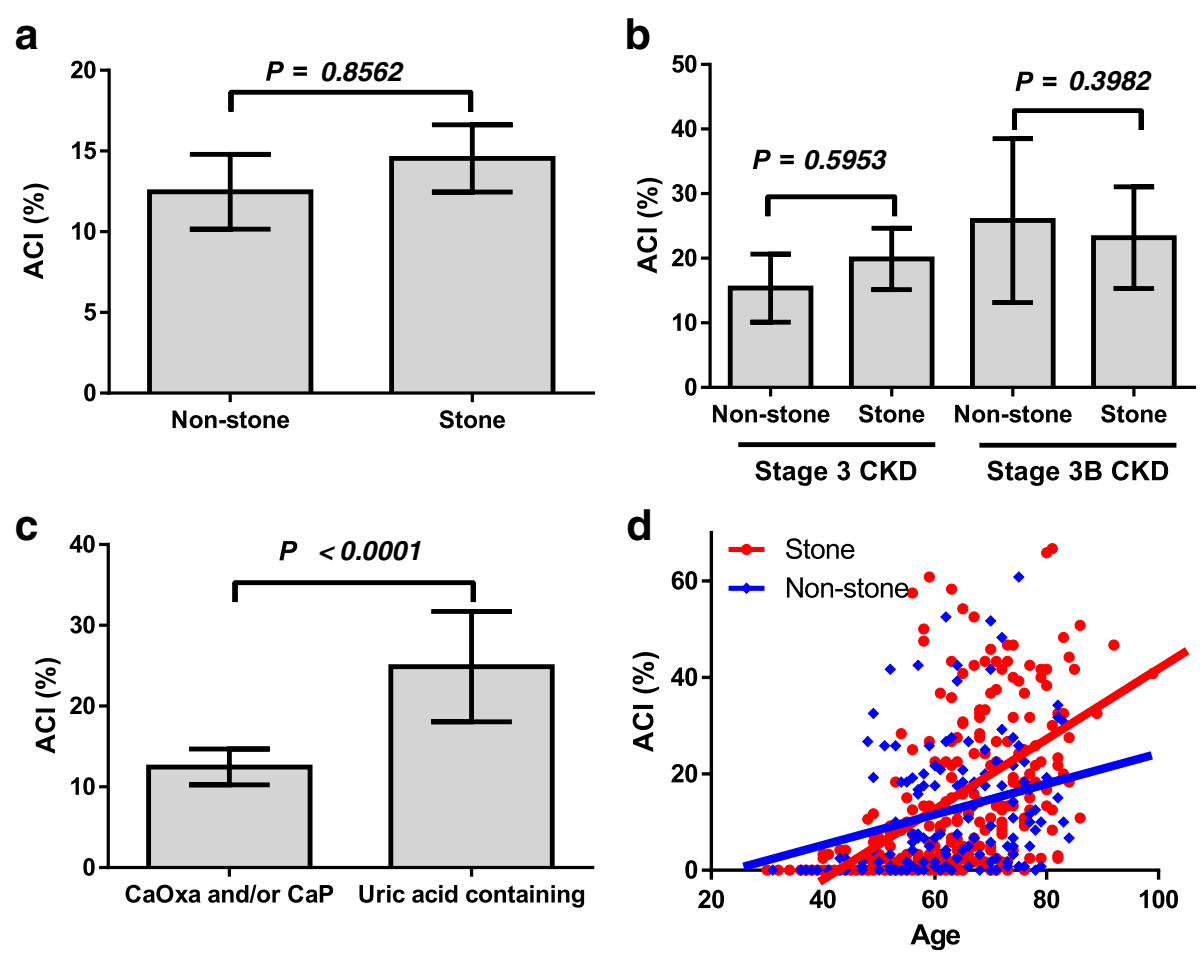

Fig. 2 Aortic calcification index (ACl) between control subjects and stone patients. ACl was not significantly different between the Non-stone and Stone groups (a). In the stage 3 or 3B CKD patients, there are no significant differences in ACl between the groups (b). ACl was significantly higher in uric acid containing stone patients (c). Scatter plot analyses are performed to compare the relationship between aortic calcification index $(\mathrm{ACl})$ and age. Linear approximations of $\mathrm{ACl}$ and age show a positive correlation in the Non-stone (blue line, $\left.\mathrm{R}^{2}=0.071, P<0.001\right)$ and Stone group (red line, $R^{2}=0.285, P<0.001$ ) (Spearman's correlation coefficient test). Age-adjusted $\mathrm{ACl}$ (a slope of line) is greater in the Stone group (0.744) compared with the Non-stone group (0.468) (d) 
Independent factors influencing stage $3 \mathrm{~B} \mathrm{CKD}$ at the time of diagnosis were identified by multivariate analyses using a logistic regression model. Odds ratios (ORs) with 95\% confidence intervals (CIs) were calculated after concurrently adjusting for potential confounders. Data from healthy volunteers were not included in this multivariate model due to the absence of ACI and proteinuria information. We included nine variables in the logistic regression analysis because of the limited number of samples. Several independent variables that increased the postoperative renal impairment risk were included in the models: age ( $>65$ years), sex (male), history of comorbidity (hypertension, type 2 diabetes, or cardiovascular disease), lipid metabolism abnormality (total cholesterol $>220 \mathrm{mg} / \mathrm{dL}$ or triglyceride $>140 \mathrm{mg} / \mathrm{dL}$ ), serum uric acid concentration $(>7.0 \mathrm{mg} / \mathrm{dL})$, voluntary urine protein $(>30 \mathrm{mg} / \mathrm{dL})$, and $\mathrm{ACI}(>13.0 \%)$ at the time of diagnosis. Moreover, the predictive accuracy of the selected variables in the dataset was evaluated using the area under the curve (AUC) derived from the ROC curve.

\section{Results}

Comparison between community-dwelling volunteers and early stage RCC patients

We compared pair-matched 296 community-dwelling volunteers and 148 of early stage RCC patients. Median age, sex, body mass index, positive history of hypertension, diabetes, and cardiovascular disease were not significantly different. In addition, unadjusted parameters such as eGFR, the number of subjects with stage $3 \mathrm{~B}$ CKD, hyperuricemia, and dyslipidemia were also not significantly different (Table 1). Based on these results, we regarded early stage RCC patients as appropriate candidate for control subjects.

Comparison between early stage RCC patients (Non-stone group) and stone patients (Stone group)

Thereafter, we compared the Non-stone and Stone groups. Component of stones were calcium oxalate $(n=119$, $41.5 \%)$, calcium oxalate mixed stones $(n=97,33.8 \%)$, uric acid containing stone $(n=31,10.8 \%)$, cysteine $(n=1$, $0.35 \%)$, and unknown $(n=39,13.6 \%)$. Table 2 summarizes
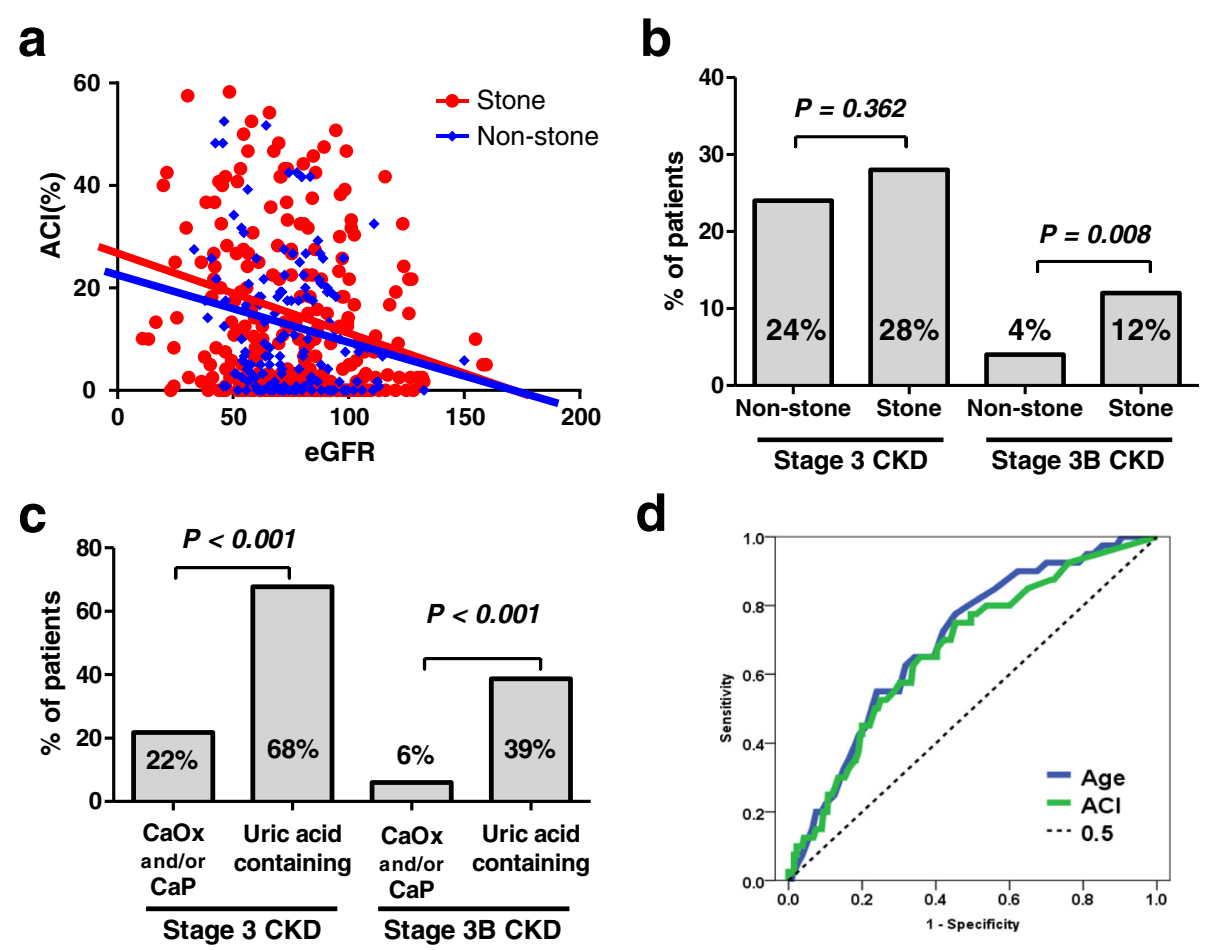

d

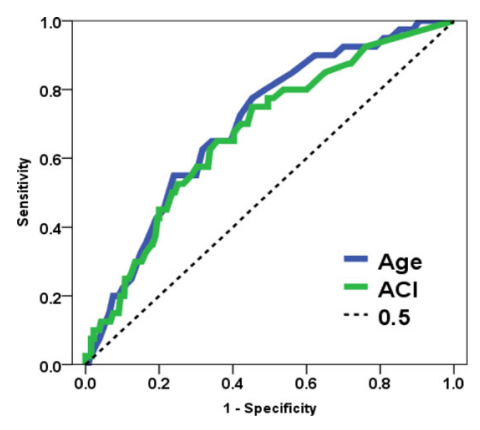

Fig. 3 Correlation eGFR and aortic calcification index (ACl), chronic kidney disease (CKD), and receiver operating characteristic curve (ROC) analysis for predictive accuracy of stage $3 \mathrm{~B}$ chronic kidney disease. ACl and eGFR showed significant, but weak correlations in the Stone $\left(R^{2}=0.053, P<0.001\right)$ and Non-stone group $\left(R^{2}=0.032, P=0.029\right)(\mathbf{a})$. The number of patients with stage $3 B$ CKD was significantly higher in the Stone group compared with the Non-stone group ( $12 \%$ vs. $4 \%, P=0.008)$, although the number of patients with stage 3 CKD was not significantly different (b). The number of patients with stage 3 and $3 \mathrm{~B}$ CKD was significantly higher in uric stone containing patients compared with calcium oxalate (CaOx) and/or calcium phosphate (CaP) stone patients (c). The optimal ACl cut-off value of age and ACl for stage 3B CKD was determined by analyzing ROC curves using the area under the curve (AUC) (d). An age of 65 years (AUC $=0.70 ; P<0.001 ; 95 \% \mathrm{Cl}: 0.62-0.78$, blue line) and $\mathrm{ACl}$ of $13.0 \%$ (AUC $=0.68 ; P<0.001 ; 95 \% \mathrm{Cl}: 0.59-0.76$, green line) were used as the cut-off values in this study 
the patient characteristics. The number of patients with hypertension was significantly higher in the Stone group $(P=0.001)$. No significant differences were observed for any other parameter. ACI was not significantly different between the groups (Fig. 2a). In addition, there were no statistical significant differences in $\mathrm{ACI}$ in patients with stage 3 CKD or stage $3 \mathrm{~B}$ CKD (Fig. 2b). Among urolithiasis patients, ACI was significantly higher in uric acid containing stone patients (Fig. 2c). Age and ACI showed positive correlations in the Stone group $\left(R^{2}=0.285, P<0.001\right)$ and Non-stone group $\left(R^{2}=0.071\right.$, $P<0.001$, Spearman's correlation coefficient test). Ageadjusted ACI (a slope of the line) was greater in the Stone group (0.744) than the Non-stone group (0.468) (Fig. 2d).

Although ACI and eGFR showed negative correlations (Fig. 3a), $\mathrm{R}^{2}$ values showed weak correlation in both groups (Stone: $R^{2}=0.053, P<0.001$, and Nonstone: $R^{2}=0.032, P=0.029$ ). The number of patients with stage 3 CKD was not significantly different between the Non-stone and Stone groups (28\% vs. 24\%, $P=0.362$ ). However, the number of patients with stage 3B CKD was significantly higher in the Stone group compared with the Non-stone group (12\% vs. $4 \%$, respectively, $P=0.008$ ) (Fig. 3b). Similarly, patients with uric acid containing stone were higher in stage 3A/3B CKD (Fig. 3c, Table 3).

To investigate the implications of ACI in renal function, the optimal ACI cut-off value for age and ACI was determined by analyzing ROC curves. An age of 65 years (AUC $=0.70, P<0.001,95 \%$ CI: $0.62-0.78$ ) and ACI of $13.0 \%$ (AUC $=0.68, P<0.001,95 \%$ CI: $0.59-0.76$ ) were used as the cut-off values in this study (Fig. 3d).

Multivariate logistic regression analysis revealed that an age of $>65$ years, sex (male), presence of comorbidities, serum uric acid $(>7.0 \mathrm{mg} / \mathrm{dL})$ were selected as independent factors for higher ACI. Similarly, an age of $>65$ years, sex (male), hyperuricemia, voluntary urine protein $>30 \mathrm{mg} / \mathrm{d}, \mathrm{ACI}>13.0 \%$, and Stone group were selected as independent factors for stage 3B CKD at the time of diagnosis (Table 4). The three group comparisons among the community-dwelling volunteers, Non-stone and Stone groups were shown in Fig. 4.

Table 3 Relationship between stage of CKD and stone components

\begin{tabular}{llll}
\hline & Uric acid (\%) & CaOx / CaP (\%) & $P$ value \\
\hline Normal / CKD 1 & $0(0 \%)$ & $70(32 \%)$ & $<0.001$ \\
CKD 2 & $9(41 \%)$ & $99(46 \%)$ & 0.078 \\
CKD 3A & $9(41 \%)$ & $34(16 \%)$ & 0.068 \\
CKD 3B & $5(19 \%)$ & $10(5 \%)$ & 0.027 \\
CKD 4 & $7(29 \%)$ & $3(1 \%)$ & $<0.001$ \\
\hline
\end{tabular}

$\mathrm{CaO}$ calcium oxalate, $\mathrm{CaP}$ Calcium phosphate
Table 4 Multivariate logistic regression analyses of independent factors for higher $\mathrm{ACl}(>13 \%)$ and stage $3 \mathrm{~B}$ CKD or higher (eGFR $<45 \mathrm{~mL} / \mathrm{min} / 1.73 \mathrm{~m}^{2}$ ) at the time of diagnosis between the Non-stone and Stone groups

\begin{tabular}{lllll}
\hline ACl & Factors & $P$ value & OR & $95 \% \mathrm{Cl}$ \\
Age & $>65$ years & 0.000 & 3.90 & $2.47-6.17$ \\
Sex & Male & 0.013 & 1.76 & $1.13-2.76$ \\
Body mass index & $>25 \mathrm{~kg} / \mathrm{m}^{2}$ & 0.132 & 0.71 & $0.46-1.11$ \\
Comorbidities & Positive & 0.049 & 1.60 & $1.00-2.55$ \\
Lipid metabolism abnormality & Positive & 0.337 & 0.80 & $0.51-1.26$ \\
Serum uric acid & $>7.0 \mathrm{mg} / \mathrm{dL}$ & 0.047 & 0.52 & $0.27-0.99$ \\
Urine protein & $>30 \mathrm{mg} / \mathrm{dL}$ & 0.089 & 1.63 & $0.93-2.87$ \\
CKD stage & $3 \mathrm{~B}$ or higher & 0.052 & 2.17 & $0.99-4.74$ \\
Stone formers & Positive & 0.501 & 0.85 & $0.54-1.35$ \\
Stage 3B CKD & Factors & $P$ value & OR & $95 \% \mathrm{Cl}$ \\
Age & $>65 \mathrm{years}$ & 0.004 & 4.11 & $1.59-10.6$ \\
Sex & Male & 0.030 & 2.56 & $1.09-5.99$ \\
Body mass index & $>25 \mathrm{~kg} / \mathrm{m}^{2}$ & 0.714 & 1.15 & $0.54-2.44$ \\
Comorbidities & Positive & 0.606 & 1.26 & $0.52-3.03$ \\
Lipid metabolism abnormality & Positive & 0.904 & 1.05 & $0.49-2.27$ \\
Serum uric acid & $>7.0 \mathrm{mg} / \mathrm{dL}$ & 0.001 & 4.53 & $1.94-10.6$ \\
Urine protein & $>30 \mathrm{mg} / \mathrm{dL}$ & 0.003 & 3.22 & $1.50-6.91$ \\
ACl & $>13.0 \%$ & 0.047 & 2.28 & $1.01-5.17$ \\
Stone formers & Positive & 0.005 & 4.00 & $1.52-10.5$ \\
\hline Comor & & &
\end{tabular}

Comorbidities included history of diabetes, hypertension, or cardiovascular disease $\mathrm{ACl}$ aortic calcification index

\section{Discussion}

Aortic calcification is regarded as one of the consequences of systemic aortic degradation. Numerous studies have addressed the clinical importance of arterial calcification among CKD patients and cardiovascular high-risk patients $[10-12,14,19-21]$. Our previous study suggested that aortic calcification burden has a negative effect on postoperative renal function in renal transplant patients, [14] and the preoperative condition of aortic calcification has a significant impact on postoperative persistent hypertension after unilateral adrenalectomy in patients with aldosterone-producing adenomas. [13] However, only a few studies have demonstrated the impact of arterial calcification in urolithiasis patients. Yasui et al. [15] reported that aortic calcification scores were significantly different between stone and non-stone patients. They also suggested that a diet with high animal protein, cholesterol, and fat may cause urolithiasis and arteriosclerosis. Therefore, we hypothesized that aortic calcification may play an important role in renal function deterioration in urolithiasis patients. However, our results suggested that ACI was not significantly higher in urolithiasis patients with CKD, whereas the age-related aortic calcification is greater in urolithiasis 


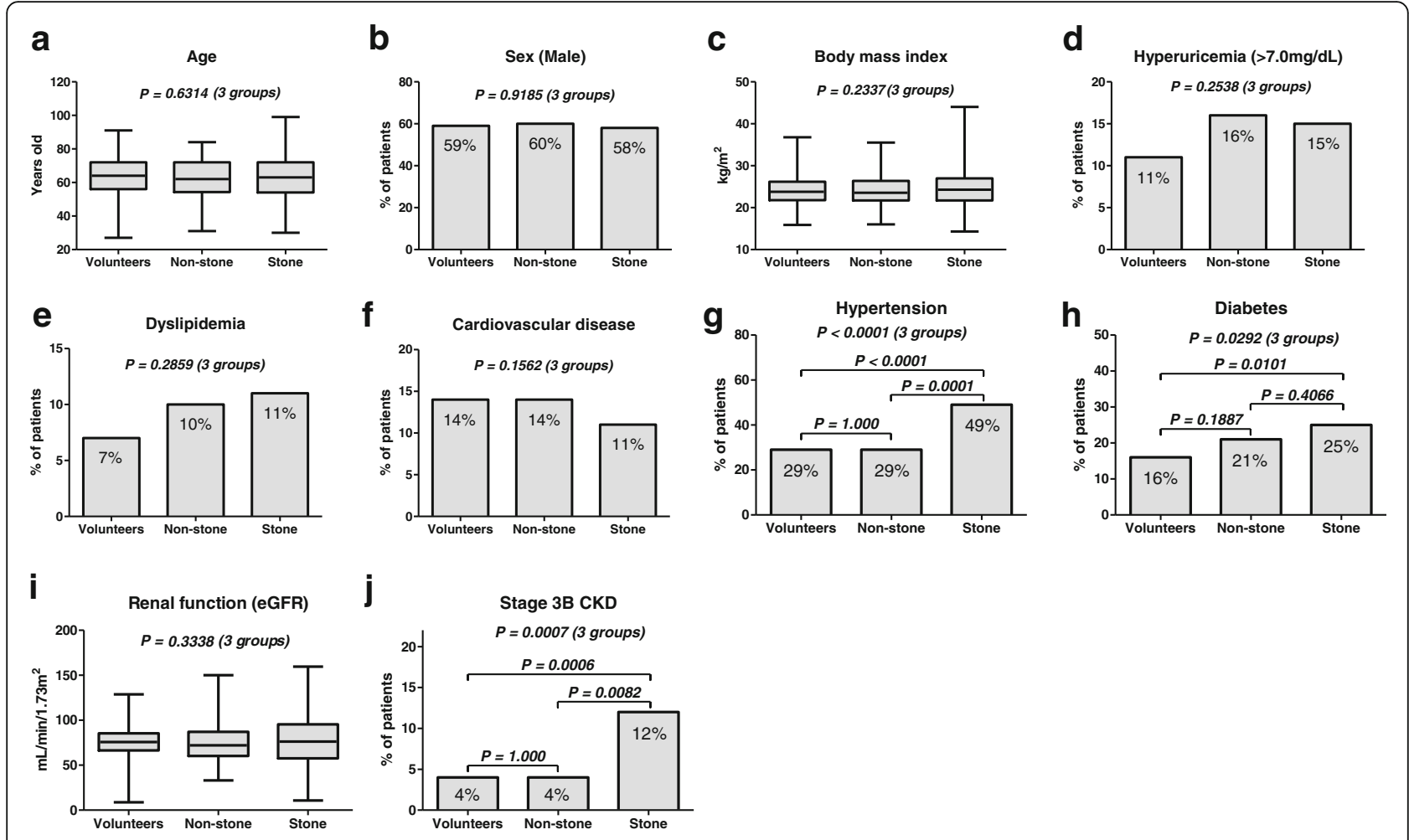

Fig. 4 The three group comparisons among the community-dwelling volunteers, Non-stone, and Stone groups. There were no significantly differences in age (a), sex (b), body mass index (c), hyperuricemia (d), dyslipidemia (e), positive history of cardiovascular disease (f) and eGFR (i), except for hypertension $(\mathbf{g})$, diabetes $(\mathbf{h})$, and prevalence of stage 3B CKD (j). It is remarkable that the prevalence of stage 3B CKD is significantly higher in the Stone group

patients. Although we could not prove the relationship between $\mathrm{ACI}$ and $\mathrm{CKD}$ in urolithiasis patients, the number of stage 3B CKD patients was significantly higher in the Stone group. The prevalence of stage 3B CKD in the community-dwelling volunteers, Non-stone, and Stone group showed $4 \%, 4 \%$, and $12 \%$, respectively (Fig. 4J). In addition, multivariate analysis identified $\mathrm{ACI}$ and being a stone former as potential factor for stage 3B CKD, which is similar to age, gender, hyperuricemia, and proteinuria. Our finding suggested that patients with poor renal function have more aortic calcification in stone formers as well as the control subjects. On the other hand, being a stone former was not selected as independent factors for higher ACI, whereas age, sex and hyperuricemia were associated with higher ACI. These results suggest a complicated relationship among vascular calcification, CKD and stone formation. Although precise mechanisms for urinary stone formation and aortic calcification remain unclear, higher age-adjusted ACI in urolithiasis patients suggested that MetS, including obesity, hypertension, impaired glucose metabolism, hyperuricemia, and atherogenic dyslipidemia, may play a key role in the vascular calcification and urolithiasis development, and resulted in premature vascular aging in stone formers.

Although mechanisms responsible for vascular calcification is still under investigation, it has been reported that vascular smooth muscle cells play a critical role in mediating vessel calcification by differentiating into osteoblast-like cells [22]. Calcifying vascular cells, which are a subpopulation of vascular smooth muscle cells, spontaneously form ossified nodules when cultured for a long time. These nodules express many bone-related molecules, including increased alkaline phosphatase activity, and osteocalcin, osteonectin, and osteopontin expressions [23]. Osteopontin is a glycoprotein secreted by macrophages, vascular smooth muscle cells, and endothelial cells and has been demonstrated to promote macrophage chemotaxis [24, 25]. It has been identified as a major matrix component of urinary calcium stones [26] and is strongly associated with urinary stone formation [27]. Recent studies have suggested that renal tubular inflammation, involving macrophages and osteopontin, plays a key role in renal calcium oxalate crystal formation [28]. Inflammation and osteopontin also play key roles in vascular calcification $[25,29]$ and inflammation, impaired calcium and phosphate homeostasis, and oxidative stress have been linked to vascular calcification in CKD patients. [30]. Furthermore, MetS has been considered as a chronic, low grade, systemic inflammatory disease [31]. Based on these results, vascular calcification and urinary stone formation might share similar mechanisms through inflammation, which 
is regarded as the key process underlying metabolic diseases. Further studies are necessary to address the detailed association between MetS, inflammation, oxidative stress, vascular calcification, urinary stone formation, and CKD.

Several limitations in this study need to be noted. The small sample size and retrospective design prevented definitive conclusions on the aortic calcification influence on renal function deterioration. We were unable to control selection bias and other unmeasurable confounding factors in both the stone and non-stone subjects even using matching methods. Although we used propensity matching methods to guarantee the validity of early stage RCC patients, adequacy of early stage RCC patients as a control subject remain unclear. We were also unable to include some other established factors that influence aortic calcification and renal function, such as cigarette smoking, information on blood pressure control, medications, and presence of hydronephrosis. We could not address the direct interaction between higher ACI and having urolithiasis because of cross-sectional study. In addition, we could not address the implication of uric acid stone on $\mathrm{ACI}$ and renal function due to the limited number of uric acid stone patients. Many statistics were also the limitation of the present study. Therefore, additional large-scale investigations are necessary to validate the ACI impact on renal function in urolithiasis patients.

Despite these limitations, the strength of this study is that it is the first report to assess the implication of aortic calcification in urolithiasis patients. Using this noninvasive modality, we could demonstrate an independent association between ACI and renal impairment in urolithiasis patients. In addition, a possible relation of ACI and hyperuricemia through MetS is suggested. Our findings may contribute for clinicians to take intensive care and educate urolithiasis patients with severe aortic calcification to prevent renal impairment progression.

\section{Conclusion}

In conclusion, aortic calcification and being a stone former had harmful influence on renal function. Premature vascular aging may be accelerated through MetS in stone formers. Further large-scale studies are needed to assess the clinical relevance of $\mathrm{ACI}$ on renal function in urolithiasis patients.

\section{Abbreviations}

ACl: Aortic calcification; AUC: Area under the curve; CaOx: Calcium oxalate; CaP: Calcium phosphate; Cls: Confidence intervals; CKD: Chronic kidney disease; CT: Computed tomography; eGFR: Estimated glomerular filtration rate; Mets: Metabolic syndrome; ORs: Odds ratios; Q1: First quartile; Q3: Third quartile; RCC: Renal cell carcinoma; ROC: Receiver operating characteristic

\section{Acknowledgement}

We thank Yuki Fujita and Mihoko Osanai for their invaluable help with the data collection.

\section{Funding}

This work was supported by a Grant-in-Aid for Scientific Research (No. 23791737, 24659708, and 22390301) from the Japan Society for the Promotion of Science.

Availability of data and materials

Because informed consents for publication of the dataset from participants are not obtained, data and materials in the present study will not be shared to protect participants' privacy and identity.

\section{Authors' contributions}

Conceived and designed the experiments: SH Performed the experiments: TT, HY, TN, TM, OS, TY (Takahiro Yoneyama), YH Analyzed the data: YT (Yuriko Terayama), TY (Tohru Yoneyama) Contributed reagents/materials/analysis tools: YT (Yuki Tobisawa), TY (Tohru Yoneyama), IT, SN Wrote the manuscript: TT, SH, IH.Other: Supervision: CO, TK, TF. All authors read and approved the final manuscript.

\section{Authors' information}

$\mathrm{TT}$ : postgraduate student, SH: assistant professor, HY: assistant professor, TN: postgraduate student, $\mathrm{H}$ : postgraduate student, TM: postgraduate student, OS: postgraduate student, YT: associate professor, TY: associate professor, TY: associate professor, YH: associate professor, TK: associate professor, IT:

associate professor, SN: professor, YT: academic researcher, TF: administrative director, CO: professor and chairman.

\section{Competing interests}

The authors declare that they have no competing interests, and no financial conflict of interest.

\section{Ethics approval and consent to participate}

The study was performed in accordance with the ethical standards of the Declaration of Helsinki and was approved by the ethical committee of Hirosaki University Graduate School of Medicine (authorization number, 2015-184). For this type of retrospective study, formal patient consent is not required. The cross sectional data collection for the Iwaki Health Promotion Project was approved by the Ethics Committee of Hirosaki University School of Medicine (authorization number, 2014-015), and all subjects provided written informed consent before participating in the project.

\section{Publisher's Note}

Springer Nature remains neutral with regard to jurisdictional claims in published maps and institutional affiliations.

\section{Author details}

'Department of Urology, Hirosaki University Graduate School of Medicine, 5 Zaifu-chou, Hirosaki 036-8562, Japan. ²Department of Advanced Transplant and Regenerative Medicine, Hirosaki University Graduate School of Medicine, Hirosaki, Japan. ${ }^{3}$ Department of Urology, Oyokyo Kidney Research Institute, Hirosaki, Japan. ${ }^{4}$ Department of Social Medicine, Hirosaki University School of Medicine, Hirosaki, Japan.

Received: 11 July 2016 Accepted: 27 March 2017

Published online: 04 April 2017

\section{References}

1. Stamatelou KK, Francis ME, Jones CA, Nyberg LM, Curhan GC. Time trends in reported prevalence of kidney stones in the United States: 1976-1994. Kidney Int. 2003;63(5):1817-23.

2. Yasui $T$, Iguchi $M$, Suzuki $S$, Kohri K. Prevalence and epidemiological characteristics of urolithiasis in Japan: national trends between 1965 and 2005. Urology. 2008;71(2):209-13.

3. Yasui T, Okada A, Hamamoto S, Hirose M, Ando R, Kubota Y, Tozawa K, Hayashi Y, Gao B, Suzuki S, et al. The association between the incidence of urolithiasis and nutrition based on Japanese National Health and Nutrition Surveys. Urolithiasis. 2013;41(3):217-24.

4. Esposito K, Chiodini P, Colao A, Lenzi A, Giugliano D. Metabolic syndrome and risk of cancer: a systematic review and meta-analysis. Diabetes Care. 2012;35(11):2402-11.

5. Kawamoto R, Kohara K, Tabara Y, Miki T. An association between metabolic syndrome and the estimated glomerular filtration rate. Internal medicine (Tokyo, Japan). 2008;47(15):1399-406. 
6. Rule AD, Krambeck AE, Lieske JC. Chronic kidney disease in kidney stone formers. Clin J Am Soc Nephrol . 2011;6(8):2069-75.

7. Thomas G, Sehgal AR, Kashyap SR, Srinivas TR, Kirwan JP, Navaneethan SD. Metabolic syndrome and kidney disease: a systematic review and metaanalysis. Clin J Am Soc Nephrol. 2011;6(10):2364-73.

8. Bahous SA, Blacher J, Safar ME. Aortic stiffness, kidney disease, and renal transplantation. Curr Hypertens Rep. 2009;11(2):98-103.

9. Walsh CR, Cupples LA, Levy D, Kiel DP, Hannan M, Wilson PW, O'Donnell CJ. Abdominal aortic calcific deposits are associated with increased risk for congestive heart failure: the Framingham Heart Study. Am Heart J. 2002;144(4):733-9.

10. Nakagami H, Osako MK, Morishita R. New concept of vascular calcification and metabolism. Curr Vasc Pharmacol. 2011;9(1):124-7.

11. Mizobuchi M, Towler D, Slatopolsky E. Vascular calcification: the killer of patients with chronic kidney disease. J Am Soc Nephrol. 2009;20(7):1453-64.

12. Tsushima M, Terayama Y, Momose A, Funyu T, Ohyama C, Hada R. Carotid intima media thickness and aortic calcification index closely relate to cerebro- and cardiovascular disorders in hemodialysis patients. Int J Urol. 2008;15(1):48-51.

13. Fujita N, Hatakeyama S, Yamamoto H, Tobisawa Y, Yoneyama T, Yoneyama T, Hashimoto Y, Koie T, Nigawara T, Ohyama C. Implication of aortic calcification on persistent hypertension after laparoscopic adrenalectomy in patients with primary aldosteronism. Int J Urol. 2016;23(5):412-7.

14. Imanishi K, Hatakeyama S, Yamamoto H, Okamoto A, Imai A, Yoneyama T, Hashimoto Y, Koie T, Fujita T, Murakami R, et al. Post-transplant renal function and cardiovascular events are closely associated with the aortic calcification index in renal transplant recipients. Transplant Proc. 2014;46(2):484-8.

15. Yasui T, Itoh Y, Bing G, Okada A, Tozawa K, Kohri K. Aortic calcification in urolithiasis patients. Scand J Urol Nephrol. 2007;41(5):419-21.

16. Imai E, Horio M, Iseki K, Yamagata K, Watanabe T, Hara S, Ura N, Kiyohara Y, Hirakata H, Moriyama T, et al. Prevalence of chronic kidney disease (CKD) in the Japanese general population predicted by the MDRD equation modified by a Japanese coefficient. Clin Exp Nephrol. 2007;11(2):156-63.

17. Satake R, Sugawara N, Sato K, Takahashi I, Nakaji S, Yasui-Furukori N, Fukuda S. Prevalence and Predictive Factors of Irritable Bowel Syndrome in a Community-dwelling Population in Japan. Intern Med. 2015;54(24):3105-12.

18. Hatakeyama S, Koie T, Narita T, Hosogoe S, Yamamoto H, Tobisawa Y, Yoneyama T, Yoneyama T, Hashimoto Y, Ohyama C. Renal Function Outcomes and Risk Factors for Risk Factors for Stage 3B Chronic Kidney Disease after Urinary Diversion in Patients with Muscle Invasive Bladder Cancer. PLoS One. 2016;11(2):e0149544.

19. Akobeng AK. Understanding diagnostic tests 3: Receiver operating characteristic curves. Acta Paediatr. 2007;96(5):644-7.

20. Li LC, Lee YT, Lee YW, Chou CA, Lee CT. Aortic arch calcification predicts the renal function progression in patients with stage 3 to 5 chronic kidney disease. Biomed Res Int. 2015;2015:131263.

21. Thomas IC, Ratigan AR, Rifkin DE, Ix JH, Criqui MH, Budoff MJ, Allison MA. The association of renal artery calcification with hypertension in community-living individuals: the multiethnic study of atherosclerosis. J Am Soc Hypertens. 2016;10(2):167-74.

22. Johnson RC, Leopold JA, Loscalzo J. Vascular calcification: pathobiological mechanisms and clinical implications. Circ Res. 2006;99(10):1044-59.

23. Watson KE, Bostrom K, Ravindranath R, Lam T, Norton B, Demer LL. TGFbeta 1 and 25-hydroxycholesterol stimulate osteoblast-like vascular cells to calcify. J Clin Invest. 1994;93(5):2106-13.

24. Sodek J, Ganss B, McKee MD. Osteopontin. Critical Reviews in Oral Biology and Medicine. 2000;11(3):279-303.

25. Gravallese EM. Osteopontin: a bridge between bone and the immune system. J Clin Invest. 2003;112(2):147-9.

26. Kohri K, Suzuki Y, Yoshida K, Yamamoto K, Amasaki N, Yamate T, Umekawa $T$, Iguchi $M$, Sinohara $H$, Kurita T. Molecular cloning and sequencing of cDNA encoding urinary stone protein, which is identical to osteopontin. Biochem Biophys Res Commun. 1992;184(2):859-64.

27. Kohri K, Nomura S, Kitamura Y, Nagata T, Yoshioka K, Iguchi M, Yamate T,

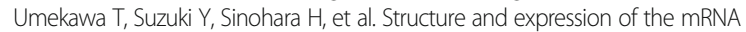
encoding urinary stone protein (osteopontin). J Biol Chem. 1993;268(20):15180-4.

28. Okada A, Yasui T, Hamamoto S, Hirose M, Kubota Y, Itoh Y, Tozawa K, Hayashi Y, Kohri K. Genome-wide analysis of genes related to kidney stone formation and elimination in the calcium oxalate nephrolithiasis model mouse: detection of stone-preventive factors and involvement of macrophage activity. J Bone Miner Res. 2009;24(5):908-24.
29. Buendia P, Montes de Oca A, Madueno JA, Merino A, Martin-Malo A, Aljama P, Ramirez R, Rodriguez M, Carracedo J. Endothelial microparticles mediate inflammation-induced vascular calcification. FASEB J. 2015;29(1):173-81.

30. Byon $\mathrm{CH}$, Chen Y. Molecular Mechanisms of Vascular Calcification in Chronic Kidney Disease: The Link between Bone and the Vasculature. Curr Osteoporos Rep. 2015;13(4):206-15.

31. Nishimura S, Manabe I, Nagasaki M, Eto K, Yamashita H, Ohsugi M, Otsu M, Hara K, Ueki K, Sugiura S, et al. CD8+ effector T cells contribute to macrophage recruitment and adipose tissue inflammation in obesity. Nat Med. 2009;15(8):914-20.

\section{Submit your next manuscript to BioMed Central and we will help you at every step:}

- We accept pre-submission inquiries

- Our selector tool helps you to find the most relevant journal

- We provide round the clock customer support

- Convenient online submission

- Thorough peer review

- Inclusion in PubMed and all major indexing services

- Maximum visibility for your research

Submit your manuscript at www.biomedcentral.com/submit

) Biomed Central 